\title{
Fast track to ion-channel modulators
}

Although ion channels are wellestablished therapeutic targets for diseases including neuropathic pain and cardiac arrhythmia, screening ion-channel modulators is relatively low-throughput and creates a bottleneck in drug discovery in this area. However, a new screening technology developed by González and colleagues could provide a muchneeded boost to drug discovery efforts.

Alterations in membrane potential govern many cellular functions and are strictly controlled by regulating ion flow through cell membrane ion channels. Conversely, opening of voltage-gated ion channels is regulated by membrane potential changes. Compounds that preferentially influence active ion channels are termed 'use-dependent' and have enhanced safety profiles; by contrast, toxins often block all channels, irrespective of activity.

Given the therapeutic potential for ion-channel modulators, demand for high-throughput screening (HTS) methods is high. The 'gold-standard' technique, whole-cell patch clamping, allows detailed characterization of channel activity but is only amenable to low-throughput screening, whereas existing HTS methods based on voltage-sensitive probes lack the temporal resolution necessary to determine a compound's mechanism of action. The authors therefore sought to combine key features of patch clamping - such as membrane potential control, repetitive stimulation and high temporal resolution
- with the high-throughput capa-

bilities of optical dyes.

In this technique, termed E-VIPR, cells in muti-well plates are subjected to electrical field stimulation using an electrode array. Fast-acting voltage-sensitive fluorescent dyes indicate changes in membrane potential, which reveals the effects of drugs on channel activity. E-VIPR was compared with patch clamping in cells expressing a human voltage-gated sodium channel, $\mathrm{hNa}_{\mathrm{v}}$ 1.3. Several known sodium-channel blockers were successfully characterized according to potency, and usedependent and non-use-dependent compounds were distinguished. Finally, 400 commonly used drugs were screened. In several cases, unexpected sodium-channel-blocking activity was demonstrated, suggesting that E-VIPR could identify compounds otherwise overlooked.

The technique is applicable to many different ion channels and could significantly advance HTS of ion-channel modulators. Further experimentation is essential, including extending its application to different cell types and channel subtypes. E-VIPR lacks the fine control of membrane potential achieved by patch clamping but has several advantages, including cost, efficiency and the capacity to identify compounds that elude patch clamping, such as slowdeveloping channel blockers. E-VIPR would probably be used to complement, rather than replace, existing methodology, facilitating the initial selection of promising compounds for more detailed investigation.

Katherine Whalley

ORIGINAL RESEARCH PAPER Huang, C. J et Characterization of voltage-gated sodiumchannel blockers by electrical stimulation and fluorescence detection of membrane potential. Nature Biotechnol. 24, 439-446 (2006)

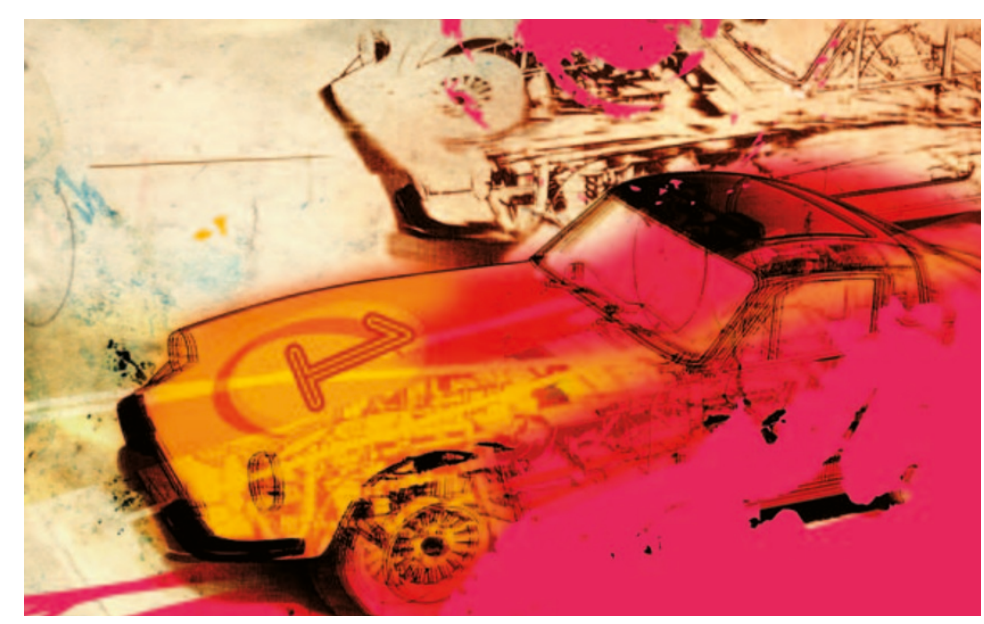

